\title{
Adaptive Thermal Management for Portable System Batteries by Forced Convection Cooling
}

\author{
Qing Xie, Siyu Yue, and Massoud Pedram \\ Department of Electrical Engineering \\ University of Southern California \\ Los Angeles, California, United States, 90089 \\ \{xqing, siyuyue, pedram\}@usc.edu
}

\author{
Donghwa Shin, and Naehyuck Chang \\ Department of EECS/CSE \\ Soule National University \\ Soule, Korea, 151-747 \\ \{dhshin, naehyuck\}@elpl.snu.ac.kr
}

\begin{abstract}
Cycle life of a battery largely varies according to the battery operating conditions, especially the battery temperature. In particular, batteries age much faster at high temperature. Extensive experiments have shown that the battery temperature varies dramatically during continuous charge or discharge process. This paper introduces a forced convection cooling technique for the batteries that power a portable system. Since the cooling fan is also powered by the same battery, it is critical to develop a highly effective, low power-consuming solution. In addition, there is a fundamental tradeoff between the service time of a battery equipped with fans and the cycle life of the same battery. In particular, as the fan speed increases, the power dissipated by the fan goes up and hence the full charge capacity of the battery is lost at a faster rate, but at the same time, the battery temperature remains lower and hence the battery longevity increases. This is the first work that formulates the adaptive thermal management problem for batteries (ATMB) in portable systems and provides a systematic solution for it. A hierarchical algorithm combining reinforcement learning at the lower level and dynamic programming at the upper level is proposed to derive the ATMB policy.
\end{abstract}

Keywords- battery system; adaptive thermal management; forced convection cooling;

\section{INTRODUCTION}

Cycle life is an important metric for the lithium ion battery that tells how many complete charge-discharge cycles it can perform before the state of health $(\mathrm{SoH})$ drops below $80 \%$. The $\mathrm{SoH}$ is defined as a figure of merit captures the general condition of the battery and its ability to store and deliver energy compared to its fresh. It generally degrades as the number of cycles, while many factors could affect the degradation rate, e.g., depth of discharge (DoD), cycling rate, and battery temperature. Elevated temperature speeds up the $\mathrm{SoH}$ degradation of the battery dramatically [1][2]. For example, the $\mathrm{SoH}$ degradation rate at $45^{\circ} \mathrm{C}$ is twice as fast as that at $35^{\circ} \mathrm{C}$ [3]. Some battery charge management policy [4] has been proposed to ensure the desired battery performance and reliability over the designed system lifetime.

The thermal behaviors of the lithium batteries has been extensively studied [5][6][7]. The experimental results in [6][7] show that the battery temperature could easily rise high $\left(>60^{\circ} \mathrm{C}\right)$ at large charge or discharge rate. This leads to serious $\mathrm{SoH}$ degradation issue. Therefore, in this paper, we propose to use properly designed and controlled forced convection cooling such as cooling fan to control the battery temperature,

This work is supported in part by a grant from the U.S. National Science Foundation, and by a grant from the BK21 Project and the NRF of Korea funded by the MEST (No. 20110030512). and thereby improve cycle life of the batteries in portable systems.

We formulate the adaptive thermal management problem for batteries (ATMB) in the portable systems with forced convection cooling as follows: how to adaptively select the fan speed so that the cumulative workload completion, defined as the cumulative amount of energy requested by the load devices over the designed system lifetime of portable systems, is maximized. The ATMB problem has two parts. First, given a multi-speed cooling fan, how to set the fan speed so that the $\mathrm{SoH}$ degradation in one cycle is minimized with a given cooling energy investment. We determine the fan speed adaptively since the heat generation and diffusion of the batteries vary from time to time.

The second part is to tradeoff the cooling energy investment and the cycle life extension. The fan speed needs to be selected very carefully, as it is also powered by the same batteries, together with the other components in the portable system. On the one hand, if we reduce the cooling investment, we will end up with little performance loss, but battery $\mathrm{SoH}$ degrades significantly due to the high temperature. On the other hand, although a high fan speed can effectively inhibit the $\mathrm{SoH}$ degradation, it comes with the price of consuming more energy, which causes higher performance loss during the current cycle. We derive the optimal global tradeoff strategy according to the designed lifetime of the portable systems.

We develop a hierarchical algorithm to determine the ATMB policy combining reinforcement learning (RL) method at the lower level and dynamic programming (DP) method at the upper level. We first obtain the tradeoff curve between the normalized fan usage and $\mathrm{SoH}$ degradation using RL method. After that, we apply DP method to determine the global tradeoff strategy for each cycle so that the cumulative workload completion is maximized for the designed system lifetime. The simulation results show that the proposed ATMB policy significantly extends the battery life by $2.08 \mathrm{X}$ and improves the cumulative workload by $70.7 \%$, comparing to the situation under natural convection.

\section{BACKGROUND}

\section{A. Related Works}

Thermal management systems for batteries using forced convection cooling have been proposed in [9][10]. Simulation results for lead-acid batteries in electrical vehicles (EVs) showed that thermal management systems of this kind cooling might improve battery performance by $30-40 \%$ [8]. However, the work on the large size lithium batteries is not applicable to the portable systems with small size batteries. Cooling fan is 
one of the most widely used forced convection cooling techniques [12], thanks to its small size, convenience, robustness, and low cost. The dynamic thermal management considering the power consumption of the cooling fan for the CPU has been studied in [11].

\section{B. Lithium Battery Thermal Behaviors}

The general thermal model of a lithium battery is very complex due to the non-uniform internal temperature distribution. The total heat generation of a lithium battery generally has two components: entropy change heat $Q_{s}$ and ohmic heat $Q_{p}[7]$,

$$
\begin{aligned}
& Q_{s}=-I T \frac{\partial V_{o c}}{\partial T}, \\
& Q_{p}=I^{2} \cdot R_{\text {int }},
\end{aligned}
$$

where $I$ is the charging or discharging current, $V_{o c}$ is the opencircuit voltage, and $R_{\text {int }}$ is the internal resistance of the lithium battery, which is not fixed but increases as SoC decreases during the discharge [7].

The energy conservation law relates the temperature change rate of the battery with the heat generation and the heat diffusion at the battery surface as,

$$
m C_{p} \frac{d T}{d t}=-h A_{e f f}\left(T-T_{a m b}\right)+Q_{s}+Q_{p},
$$

where the $m$ is battery mass, $C_{p}$ is the specific heat capacity $\left(J \mathrm{~kg}^{-1} \mathrm{~K}^{-1}\right)$ of the lithium battery, $A_{e f f}$ is the effective surface area of the lithium battery, $h$ is the heat transfer coefficient $\left(W m^{-2} K^{-1}\right)$ between the battery surface and air, $T$ is the battery cell temperature, and $T_{a m b}$ is the ambient temperature. The accepted value of $h$ for small size lithium battery is about $5 \sim 10 \mathrm{Wm}^{-2} \mathrm{~K}^{-1}$ [6][7].

\section{Forced Convection Cooling Mechanism}

In this work, we modify the original two-term thermal resistance model [12] and assume two thermal resistors connected in parallel: one natural convection resistor and one forced convection resistor (relates to the fan). Thus, Equation (2) becomes,

$$
\begin{gathered}
m C_{p} \frac{d T}{d t}=-\frac{\left(T-T_{a m b}\right)}{R_{t h}}+Q_{s}+Q_{p}, \\
R_{t h}=\left(h A_{\text {eff }}\right)^{-1} \|\left(2 \dot{m} c_{a p}\right)^{-1},
\end{gathered}
$$

where $c_{a p}$ is the specific heat capacity of the air and $\dot{m}$ is the mass flow rate of the air, depending on the fan speed and the shape of air channel. The energy consumed by the fan has a super-linear relation with respect to the fan speed [11].

\section{State-of-Health Degradation Model}

The SoH degradation rate of lithium battery depends on the cycle numbers, DoD, average SoC, and temperature [13]. The per cycle $\mathrm{SoH}$ degradation is given by,

$$
L(T)=L_{0}\left(D o D, S o C_{a v g}, S o H\right) \cdot \exp \left(\frac{U_{0}}{k} \cdot \frac{T-T_{r e f}}{T T_{r e f}}\right),
$$

where $L_{0}\left(D o D, S o C_{a v g}, S o H\right)$ is the per cycle $\mathrm{SoH}$ degradation at the reference battery temperature (normally at $25^{\circ} \mathrm{C}$ ), $k$ is the Boltzmann constant, and $U_{0}$ is the activation energy. The value of activation energy for small size lithium battery varies from 48 to $55 \mathrm{~kJ} / \mathrm{mol}$ [3], depending on the SoC.

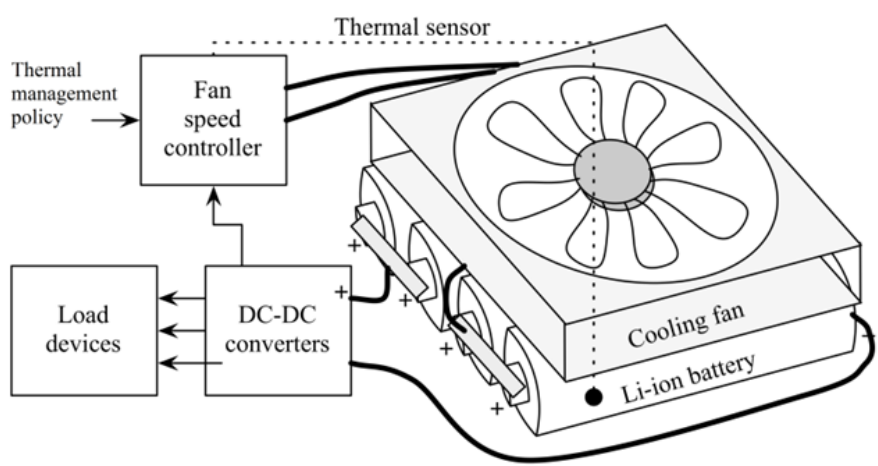

Figure 1. Structure of the proposed forced convection cooling for batteries.

\section{PROBLEM STATEMENT}

The proposed structure of a target portable system with forced convection cooling is shown in Figure 1. The system contains a battery pack as the power source, load devices, a cooling fan, which is also powered by the same battery pack, and a DC-DC converter to match with that of the load devices and the fan. We assume that the model of load demand profile is known. The fan has multiple speed levels, which consume different amount of power. The thermal management policy operates the fan speed controller and determines when to turn on/off the fan and which fan speed level to select. The load devices also generate heat during the usage, however, that is out of the scope of this work. Without loss of generality, we discuss the discharge process in this paper.

The designed system lifetime plays an important role in deriving the global tradeoff strategy between the cooling energy investment and battery cycle life extension. For relatively short lifetime, we may be able to discharge the batteries aggressively, i.e., without investing too much energy in cooling. This hurts the cycle life of the batteries largely, but minimizes the short term performance loss. In contrast, for relatively long designed lifetime, we should invest more energy in cooling, i.e., using high fan speed, as it alleviates the $\mathrm{SoH}$ degradation of batteries and extends the battery cycle life.

In this work, we define the workload completion as the energy requested by the load devices at downstream of DC-DC converter. We use cumulative workload completion (CWC) over the designed system lifetime $T_{d s l}$ as the metric function, which captures both the short term performance and long term cycle life. $T_{d s l}$ could vary from months to years, depending on the design specifications. Once the SoH drops below a threshold value, $\delta$, the batteries are assumed to reach their end of life and cannot serve the load demand any more. The well accepted value of $\delta$ is $80 \%$. Thus, we denote the cumulative workload completion by $W\left(T_{d s l}, \delta\right)$ and formulate the ATMB problem as follows:

Given: battery pack specification $\left[V_{o c}(S o C), R_{i n t}(S o C)\right.$, Size, $\left.C_{p}, h\right]$, fan specification $\quad\left[\right.$ Size, speed $\left[F_{0}, F_{1}, \ldots\right]$, power $\left.\left[P_{F 0}, P_{F 1}, \ldots\right]\right]$, load profile distribution $P_{\text {load }}(t)$;

Find: fan speed over the time, $F(t) \in\left[F_{0}, F_{1}, \ldots\right], t \in$ $\left[0, \tau_{n}\right], n \in[1, \ldots, N], F_{0}$ is the off-state of the fan and $\tau_{n}$ is the stopping time for $n$-th discharge process;

Maximize: $W\left(T_{d s l}, \delta\right)=\sum_{n=1}^{N} \int_{t=0}^{\tau_{n}} P_{\text {load }}(t) d t$, where $\mathrm{N}$ is the largest cycle index number such that $\sum_{n=1}^{N} \tau_{n} \leq T_{d s l}$; Subject to: 
(1) $\sum_{n=1}^{N} \Delta \operatorname{SoH}_{f c}(n) \leq \delta$, the subscription $f c$ stands for full cycle $\mathrm{SoH}$ degradation;

(2) $P_{\text {load }}(t)+P_{F(t)} \leq P_{B, \max }, t \in\left[0, \tau_{n}\right], n \in[1, \ldots, N]$, where $P_{B, \max }$ is the battery power capability.

\section{METHOD}

The ATMB problem in Section III is generally non-convex and thereby cannot be optimally solved. We propose an algorithm to derive the ATMB policy hierarchically. We focus on minimizing the $\mathrm{SoH}$ degradation with given amount of cooling energy investment at the lower level using Reinforcement Learning technique. At the upper level, we implement the Dynamic Programming (DP) method to derive the optimal tradeoff strategy by properly selecting the tradeoff options for each single cycle, aiming to maximize $W\left(T_{d s l}, \delta\right)$.

\section{A. Lower Level: Deriving Tradeoff Curve using $R L$}

The ATMB policy should take into account of the battery temperature, battery SoC and load demand. Thus, we divide the three terms above into a series of discrete levels and define the state set as $S=\{T\} \times\{S o C\} \times\left\{P_{\text {load }}\right\}$. We define the action set as the fan speed levels, and the state transition is calculated using Equation (1) (3). We break the entire discharge process into a series of time slots $\left\{t_{1}, t_{2}, \ldots, t_{K}\right\}$. At the beginning of $k$ th time slot in $n$-th cycle, we observe the state information $s_{k}$, pick the proper action $a_{k}$ according to cumulative state-action pairs value $\{Q(s, a)\}$. Taking action $a_{k}$ results in $\Delta \operatorname{SoH}\left(a_{k}\right)$ amount of SoH degradation and extra $P_{a_{k}}$ amount of fan power consumption. Thus, we calculate the penalty function at the end of the k-th slot as follows,

$R\left(s_{k}, a_{k}\right)=\int_{t_{k-1}}^{t_{k}} \lambda \frac{P_{a_{k}}}{E_{\text {tot }}(n)} d t+(1-\lambda) \frac{\Delta \operatorname{SoH}\left(a_{k}\right)-\Delta \operatorname{SoH}\left(F_{0}\right)}{\Delta \operatorname{SoH}_{f c}\left(n, T_{r e f}\right)}$,

where the $E_{\text {tot }}(n)$ is total energy capacity of the battery pack in $n$-th cycle, $\Delta \operatorname{SoH}_{f c}\left(n, T_{r e f}\right)$ is the $\mathrm{SoH}$ degradation over $n$-th full cycle at reference temperature $\left(25^{\circ} \mathrm{C}\right), \triangle \mathrm{SoH}\left(F_{0}\right)$ is the SoH degradation over the $k$-th time slot while fan is off. $\lambda$ is a relative weight factor, which is used to tradeoff the $\mathrm{SoH}$ degradation and fan usage. We update the $Q\left(s_{k}, a_{k}\right)$ as follows,

$$
\begin{aligned}
& Q\left(s_{k}, a_{k}\right) \leftarrow Q\left(s_{k}, a_{k}\right)+ \\
& \quad \alpha\left(R\left(s_{k}, a_{k}\right)+\beta \min _{a_{k+1}}\left(Q\left(s_{k+1}, a_{k+1}\right)\right)-Q\left(s_{k}, a_{k}\right)\right),
\end{aligned}
$$

where $\alpha$ is the learning rate and $\beta$ is the discount factor.

We achieve a tradeoff curve between the fan usage and $\mathrm{SoH}$ degradation by tuning the parameter $\lambda$, as shown in Figure 2 . Since the $\mathrm{SoH}$ degradation depends on the current $\mathrm{SoH}$ of the battery, we define the normalized tradeoff variables as,

$$
\begin{gathered}
E_{f a n}^{\text {norm }}=\sum_{k=1}^{M} \int_{t_{k-1}}^{t_{k}} P_{a_{k}} d t / E_{\text {tot }}(n) \times 100 \%, \\
\Delta \operatorname{SoH}^{\text {norm }}=\Delta \operatorname{SoH}_{f c}(n) / \operatorname{SoH}(n) \times 100 \%,
\end{gathered}
$$

where $\Delta \operatorname{SoH}_{f c}(n)$ is the total SoH loss in $n$-th cycle after applying the RL method, and $\operatorname{SoH}(n)$ is the $\mathrm{SoH}$ at that time. The state-action pairs $\{Q(s, a)\}$ and normalized variables are stored in LUTs for the upper level of algorithm.

\section{B. Upper Level: Maximizing Workload using DP}

The problem at the upper level is how to determine the optimal tradeoff strategy to meet the desired system lifetime as well as maximize the CWC. More precisely, for each cycle, we solve a sub-problem of how to pick up a tradeoff option from the lower level RL results, with a global consideration of performance and $\mathrm{SoH}$ degradation. We implement a bottom-up DP to solve this problem.

An important issue for the DP is to control the time complexity and the memory complexity. Suppose the lower level RL gives $Z$ number of tradeoff options: ( $E_{f a n, 1}^{\text {norm }}$, $\left.\Delta \mathrm{SoH}_{1}^{\text {norm }}\right),\left(E_{\text {fan }, 2}^{\text {norm }}, \Delta \mathrm{SoH}_{2}^{\text {norm }}\right), \ldots,\left(E_{\text {fan }, Z}^{\text {norm }}, \Delta \mathrm{SoH}_{Z}^{\text {norm }}\right)$, then we need try at most $Z$ tradeoff options to derive new element from each known $W(n, m)$. Thus, the total time complexity is $O(M N Z)$. The memory complexity is $O(M N)$. The SoH degradation is a continuous process so that we round it to the closest quantized SoH level during the DP. We quantize $1 \% \mathrm{SoH}$ degradation into 2000 levels during our simulation, considering both the computation precision and time complexity. The DP method is described as Algorithm 1. It finds the optimal tradeoff strategy to maximize $W(N, \Delta L)$.

Algorithm 1: Tradeoff Strategy Determination

Initialize: empty matrix $W(N, M), \operatorname{Path}(N, M)$;

For $Z$ from 1 to $Z$ :

$$
W\left(1, Q \text { uantize }\left(\Delta \operatorname{SoH}_{z}^{\text {norm }}\right)\right) \leftarrow \operatorname{CalcWL}\left(\left(E_{f a n, z}^{\text {norm }}, \Delta \operatorname{SoH}_{z}^{\text {norm }}\right)\right) \text {; }
$$

For $n$ from 1 to $N-1$ :

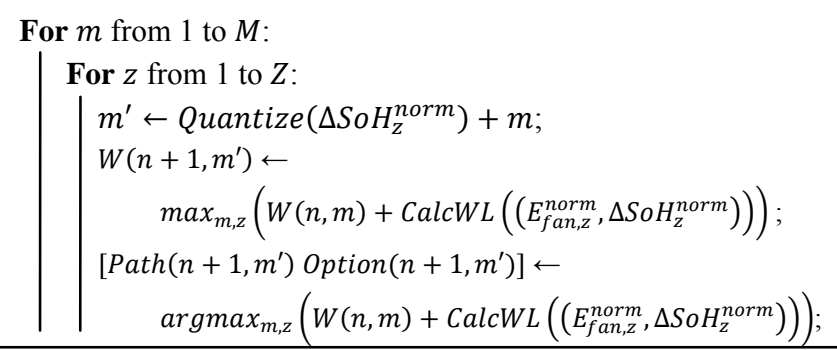

\section{RESULTS AND DISCUSSIONS}

\section{A. Simulation Setups}

We use four LiMnNi 22650M cylinder lithium battery cells as the power source and a $70 \mathrm{~mm}$ fan for the simulation. The single battery cell has height of $65 \mathrm{~mm}$, diameter of $22 \mathrm{~mm}$, weight of 60 gram, and nominal capacity of $2 \mathrm{Ah}$. The total energy capacity of the battery pack is $30.4 \mathrm{Wh}$. We target the load device as a laptop and simulate the load demands profile as a continuous profile while the magnitude of the power demand follows a normal distribution, based on the power measurement in [14]. The typical specific heat capacity of the lithium battery is around $0.8 \sim 0.9 \mathrm{~J}(\mathrm{gK})^{-1}$. We borrow the relation of internal resistance and activation energy versus battery SoC from [7]. We use a modified fan model based on [11] and assume the fan has four speed options: off (0 RPM), low (1000 RPM), medium (2000 RPM), and high (3000 RPM). We set four baseline thermal management policies using fixed fan speed. Both baseline setups and proposed policy are simulated for same battery pack mentioned above.

\section{B. Tradeoff Curve}

We apply RL and tune the weight factor $\lambda$ to change the relative weight between the fan usage and $\mathrm{SoH}$ degradation. Figure 2 shows the tradeoff curve obtained through the learning method. Each circle/triangle/square mark corresponds to one $\lambda$ setting at different $\mathrm{SoH}$ of the battery. The cross marks show the results of fan usage and $\mathrm{SoH}$ degradation applying the fixed 
fan speed. Figure 2 shows that although the battery SoH are different, the proposed normalized fan usage and $\mathrm{SoH}$ degradation have a very similar tradeoff curve. Thus, we sweep $\lambda$ values one time at $100 \% \mathrm{SoH}$ and record the tradeoff options.

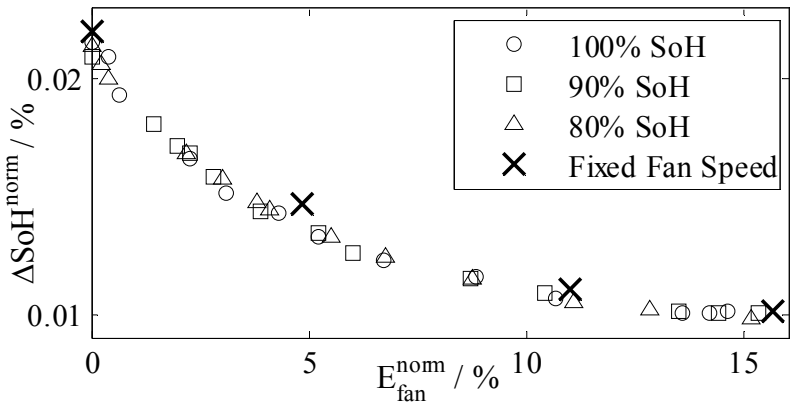

Figure 2. Tradeoff curve between the normalized fan usage and normalized $\mathrm{SoH}$ degradation.

The RL method returns the best tradeoff options it can achieve, which normally requires to adaptively set the fan speed due to the variation of actual battery temperature, $\mathrm{SoC}$, and load demand intensity. The proposed RL method shows a maximum improvement of $17.2 \%$ and $5.5 \%$ in terms of normalized fan usage and normalized $\mathrm{SoH}$ degradation.

\section{Cycle Life Extension}

We apply DP method to find the maximum CWC that the portable system can serve over the designed system lifetime. We assume that the battery reaches the end of life after its $\mathrm{SoH}$ drops below $80 \%$. Table 1 shows battery cycle life when applying different thermal management policies. Compared to the natural convection cooling, the proposed ATMB policy can extend the battery cycle life by $2.08 \mathrm{X}$, from 1065 cycles to 2215 cycles, and improve the maximum CWC by $70.7 \%$.

Table 1. Maximum cycle life and corresponding CWC that the battery can achieve by different thermal management policy.

\begin{tabular}{|c|c|c|c|c|c|}
\hline & $\begin{array}{c}\text { proposed } \\
\text { ATMB }\end{array}$ & $\begin{array}{c}\text { Fan } \\
\text { Off }\end{array}$ & $\begin{array}{c}\text { Fan } \\
\text { Low }\end{array}$ & $\begin{array}{c}\text { Fan } \\
\text { Med }\end{array}$ & $\begin{array}{c}\text { Fan } \\
\text { High }\end{array}$ \\
\hline $\begin{array}{c}\text { cycle life } \\
\text { (cycles) }\end{array}$ & 2215 & 1065 & 1479 & 2024 & 2212 \\
\hline $\begin{array}{c}\text { max CWC } \\
(\mathrm{kWh})\end{array}$ & 37.17 & 22.04 & 28.78 & 35.79 & 36.12 \\
\hline
\end{tabular}

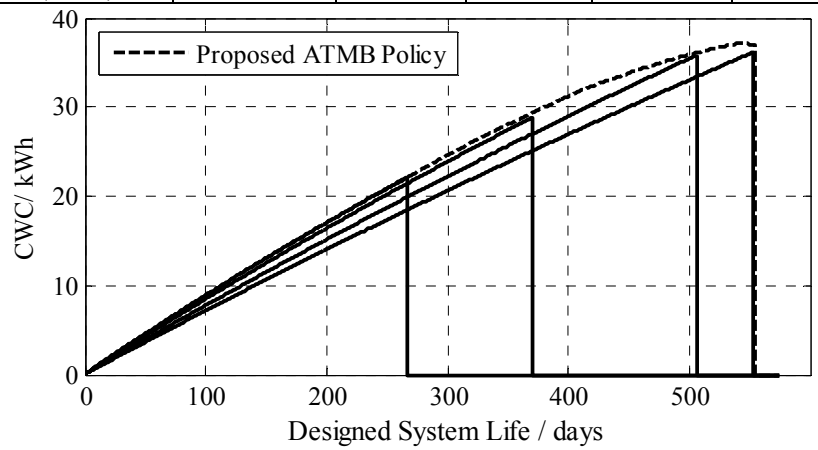

Figure 3. CWC using different thermal management policies versus designed system lifetime.

The proposed ATMB policy also shows advantage comparing to other fixed-fan speed thermal management policies. The proposed ATMB improves of cycle life of $49.8 \%$ and $9.4 \%$, and the CWC of $29.2 \%$ and $3.9 \%$, against the fanlow and fan-medium, respectively. Although the fan-high policy also effectively extends the battery cycle life, the proposed ATMB still outperforms by $2.9 \%$ in CWC.

\section{CWC versus Designed System Lifetime}

Figure 3 shows the simulation results of CWC at different thermal management policies and designed system lifetimes. The proposed ATMB not only achieves the same performance as that of natural convection cooling for relatively shorter designed system lifetime, but also extends the battery cycle life so that longer designed system lifetime is also achievable. Compared to fan-high and fan-medium policies, the proposed policy improves the CWC for the same designed system lifetime by up to $8.7 \%$ to $21.4 \%$.

\section{CONCLUSION}

This is the first paper that proposed a forced convection cooling for the batteries in portable systems and formulated a corresponding adaptive thermal management problem for batteries (ATMB). The objective of the ATMB problem is to develop an ATMB policy that maximizes the cumulative workload completion and meets the design specifications. We developed a hierarchical algorithm to derive the ATMB policy. We applied the reinforcement learning method to obtain the tradeoff curve between the fan usage and $\mathrm{SoH}$ degradation at the lower level, taking into account of battery temperature, battery state-of-charge, and load demand. At the upper level, we implemented a dynamic programming method to globally carry out the optimal tradeoff strategy for each cycle. The simulation results showed that the proposed ATMB policy extends the battery cycle life by $2.08 \mathrm{X}$ and improves the cumulative workload completion against the natural convection cooling by $70.7 \%$.

\section{REFERENCES}

[1] J. Vetter et al, "Ageing mechanisms in lithium-ion batteries," in J. of Power Sources, vol. 147, pp. 269-281, 2005.

[2] P. Ramadass, B. Haran, R. Wright, and B. Popov, "Mathematical modeling of the capacity fade of Li-ion battery", in J. of Power Sources, vol. 123, pp230-240, 2003.

[3] B. Y. Liaw et al, "Correlation of Arrhenius behaviors in power and capacity fades with cell impedance and heat generation in cylindrical lithium-ion cells", in JPS, vol. 119-121, pp. 874-886, 2003.

[4] Q. Xie et. al, "State of health aware charge management in hybrid electrical energy storage systems", in DATE, 2012.

[5] U. S. Kim, J. Yi, C. B. Shin, T. Han, and S. Park, "Modeling the thermal behavior of a lithium-ion battery during charge", in J of Power Sources, vol. 196, pp 5115-5121, 2011.

[6] Y. Ye, Y. Shi, N. Cai, J. Lee, and X. He, "Electro-thermal modeling and experimental validation for lithium ion battery", in J of Power Sources, vol. 199, pp. 227-238, 2012.

[7] K. Onda et al, "Thermal behavior of small lithium-ion battery during rapid charge and discharge cycles", in J. of Power Sources, vol.158,

[8] 300๙: Hallaj, H. Maleki, J. S. Hong, and J. R. Selman, "Thermal modeling and design considerations of lithium-ion batteries", in J. of Power Sources, vol. 83, pp. 1-8, 1999.

[9] J. Garche, and A. Jossen, "Battery management systems (BMS) for increasing battery life time", in TELESCON, 2000.

[10] K. Oshida, and S. Iwatsuki, US patent 5585204, 1996.

[11] D. Shin, S. W. Chung, E. Y. Chung, and N. Chang, "Energy-Optimal Dynamic Thermal Management: Computation and Cooling Power CoOptimization", in IEEE T. Industrial Informatics, vol. 6, 2010.

[12] R. Moffat, "Modeling Air-Cooled Heat sinks as Heat Exchangers", in IEEE Semi-therm Symposium, 2007.

[13] A. Millner, "Modeling Lithium Ion Battery Degradation in Electric Vehicles," in CITRES, 2010.

[14] A. Mahesri, and V. Vardhan, "Power Consumption Breakdown on a Modern Laptop", in PACS, 2004. 
